Appl. Ent. Zool. 15 (4) : 458-464 (1980)

\title{
Population Dynamics of Bursaphelenchus lignicolus (Nematoda: Aphelenchoididae) and B. mucronatus in Pine Seedlings ${ }^{1}$
}

\author{
Kazuyoshi FutaI \\ Laboratory of Applied Botany, Faculty of Agriculture, Kyoto University, Kyoto 606, Japan
}

(Received July 8, 1980)

\begin{abstract}
Two species of Bursaphelenchus nematode which were different in their pathogenicity to pine trees were inoculated onto the seedlings of Pinus thunbergii and $P$. densiflora, and dispersion and population density of both nematodes in the inoculated seedlings were examined once a week for 8 weeks. Pathogenic B. lignicolus dispersed and propagated rapidly in all inoculated seedlings of $P$. thunbergii but failed to disperse and propagate in some of the inoculated seedlings of $P$. densiflora. On the other hand, non-pathogenic $B$. mucronatus hardly propagated in both pines except in a few seedlings though nematodes of this species could disperse as well as $B$. lignicolus. Rapid dispersion accompanied with propagation of nematodes always resulted in weakening or death of hosts irrespective of the species of pine and nematode examined, though a few dead seedlings without so many nematodes were observed $6-8$ weeks after inoculation.
\end{abstract}

\section{INTRODUGTION}

In order to compare the resistance of a particular plant to different species of nematodes, or to compare the pathogenicity of a particular species of nematode to different plants, the rate of reproduction of the nematode has been examined with many nematode species (Jones, 1956; SeInhorst, 1967; VANGundy and Kirkpatrick, 1964). Population growth of Bursaphelenchus lignicolus, the causal agent of the wilting disease of pines, in seedlings of Pinus thunbergii and $P$. densiflora has been studied by MamiYa (1974) in relation to the water content of the hosts. Dispersion of inoculated $B$. lignicolus in wood of $P$. thunbergii, $P$. densiflora or in $P$. taeda was also investigated in connection with the reduction of oleoresin exudation which is a well known symptom of the tree diseased by B. lignicolus (Наsнimoto and Dosono, 1975). In the present investigation, the population growth and the distribution pattern of $B$. lignicolus in seedlings of $P$. thunbergii or $P$. densiflora were compared with those of $B$. mucronatus, a non-pathogenic nematode closely related to $B$. lignicolus.

\section{MATERIALS AND METHODS}

Three-year-old seedlings ( $P$. thunbergii and $P$. densiftora) were inoculated with $B$. lignicolus or $B$. mucronatus in the following manner. After peeling a small part of the

1 The main points of this paper were presented at the 80 th annual meeting of the Japanese Forestry Society, 1979. 
bark 3 to $6 \mathrm{~cm}$ from the base of the stem (about $5 \mathrm{~mm}$ wide and $3 \mathrm{~cm}$ long), a piece of absorbent cotton was placed on the wound, and a water suspension of nematdoes, 2,000 per seedling was poured on it. The nematodes which were reared on Botrytis cinerea, extracted with a Baermann funnel, and filtered through a 325 mesh to remove small individuals were used as the inocula. The nematode inoculation was conducted on the 19th of June, 1978 using 60 seedlings for each host-parasite combination, and 5 seedlings of both species inoculated with either nematode were harvested once a week for 8 weeks. After removing leaves, twigs and roots, the surface of the stem was washed, and wiped with tissue paper. The part of the stem $15 \mathrm{~cm}$ from the base was weighed for its fresh weight and cut into five short pieces each $3 \mathrm{~cm}$ long. The dry weight and the water content of these pieces were calculated from the fresh to dry weight ratio of the remaining part of the stem, on the assumption that the water content at any part of the stem was the same. Every short piece was cut longitudinally into halves. One-half was soaked in 0.5\% 2,3,5-triphenyltetrazolium chloride (TTC) solution for 12 hours, and the other half set on a Baermann funnel for 24 hours to extract nematodes. The number of nematodes recovered was counted for each half piece. Using the numbers of nematodes thus counted for five stem pieces, the population density of both pine wood nematodes was calculated for the gram dry weight of each host stem. The viability of the stem was determined from the stainability to TTC (PARKER, 1957). Furthermore, changes in the appearance of inoculated seedlings were observed every three to seven days for 8 weeks with all seedlings not yet served for the experiments on the nematode population and distribution, and the results are shown in Fig. 1 as the percentage of the weakened or dead seedlings to all remainders.

The nematodes used in the present experiments were the same as those mentioned in my previous report (FutaI, 1980).

\section{RESULTS}

\section{Symptoms of inoculated seedlings}

As shown in Fig. 1, the inoculation of $B$. lignicolus caused more rapid damages in seedlings of $P$. thunbergii than in those of $P$. densiflora. That is, complete death occurred 49 days after inoculation in $P$. thunbergii but even 57 days after inoculation some seedlings of $P$. densiflora were still alive. On the other hand, the inoculation of $B$. mucronatus caused far less damages in both pine species, i.e. only four seedlings weakened or died in $P$. thunbergii, no symptoms appeared in $P$. densiflora except for one seedling weakened to death (so the results of the latter host-parasite combination was excluded from Fig. 1).

\section{Dispersion and population growth of nematodes in pine stems}

Figure 2 illustrates the dispersion pattern of nematodes in the inoculated seedlings. The extent of damage in each seedling examined was judged from appearance and by 'TTC tissue stainability and represented by ' $W$ ' (= weakened) or ' $D$ ' (= dead) on each column in Fig. 2. The population density of $B$. lignicolus and B. mucronatus in host stem $15 \mathrm{~cm}$ long was plotted against the time after inoculation (Fig. 3a) in relation to the changes of water content in the stem (Fig. 3b).

When $B$. lignicolus was inoculated onto $P$. thunbergii every seedling was damaged to death, and such host weakness or death was always accompanied with the rapid 

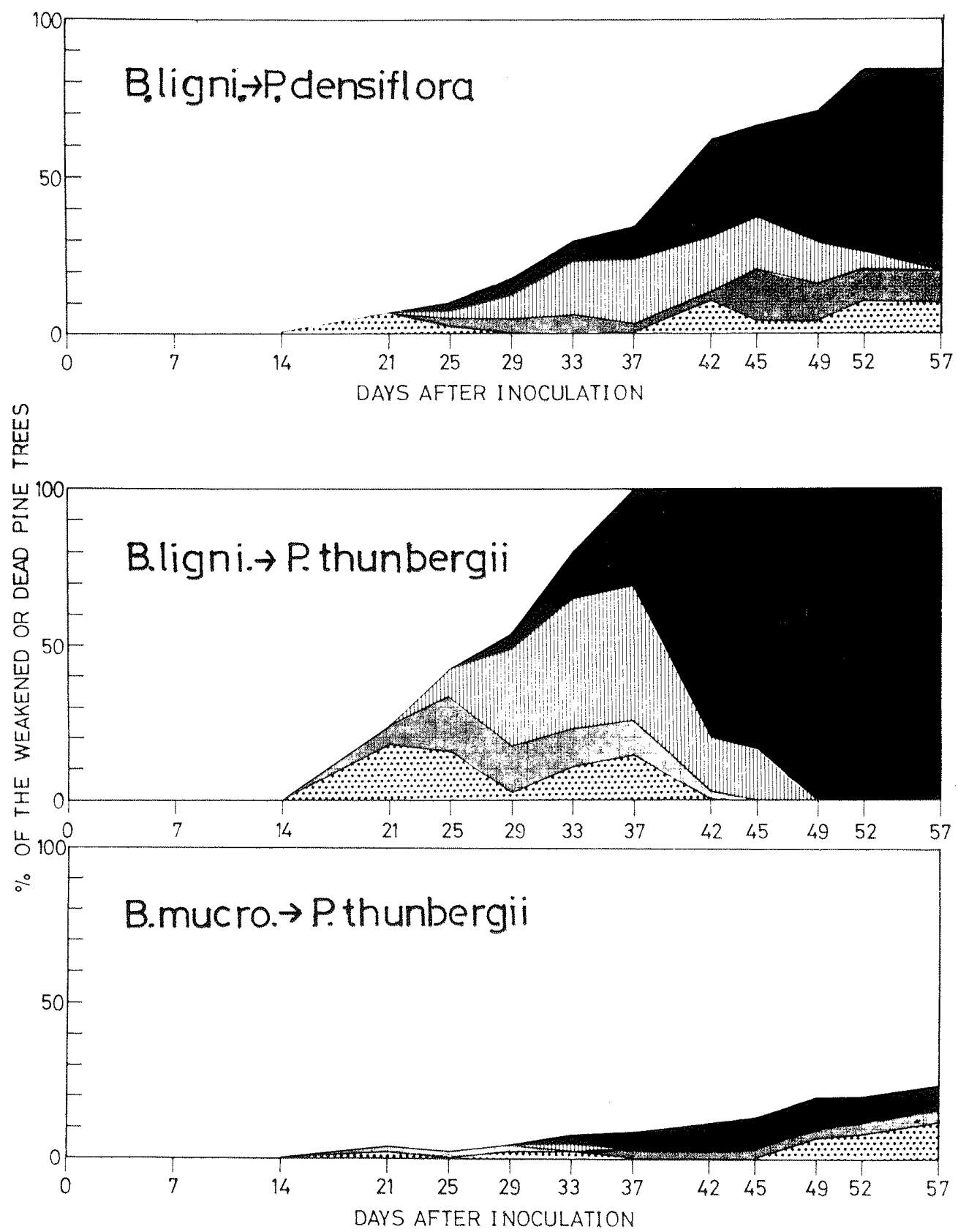

Fig. 1. Changes in percentage of the weakened or dead prine trees after inoculation of $B$. lignicolus and B. mucronatus.

dead [III : leaves are almost browned

a whole : leaves are vaguely faded 


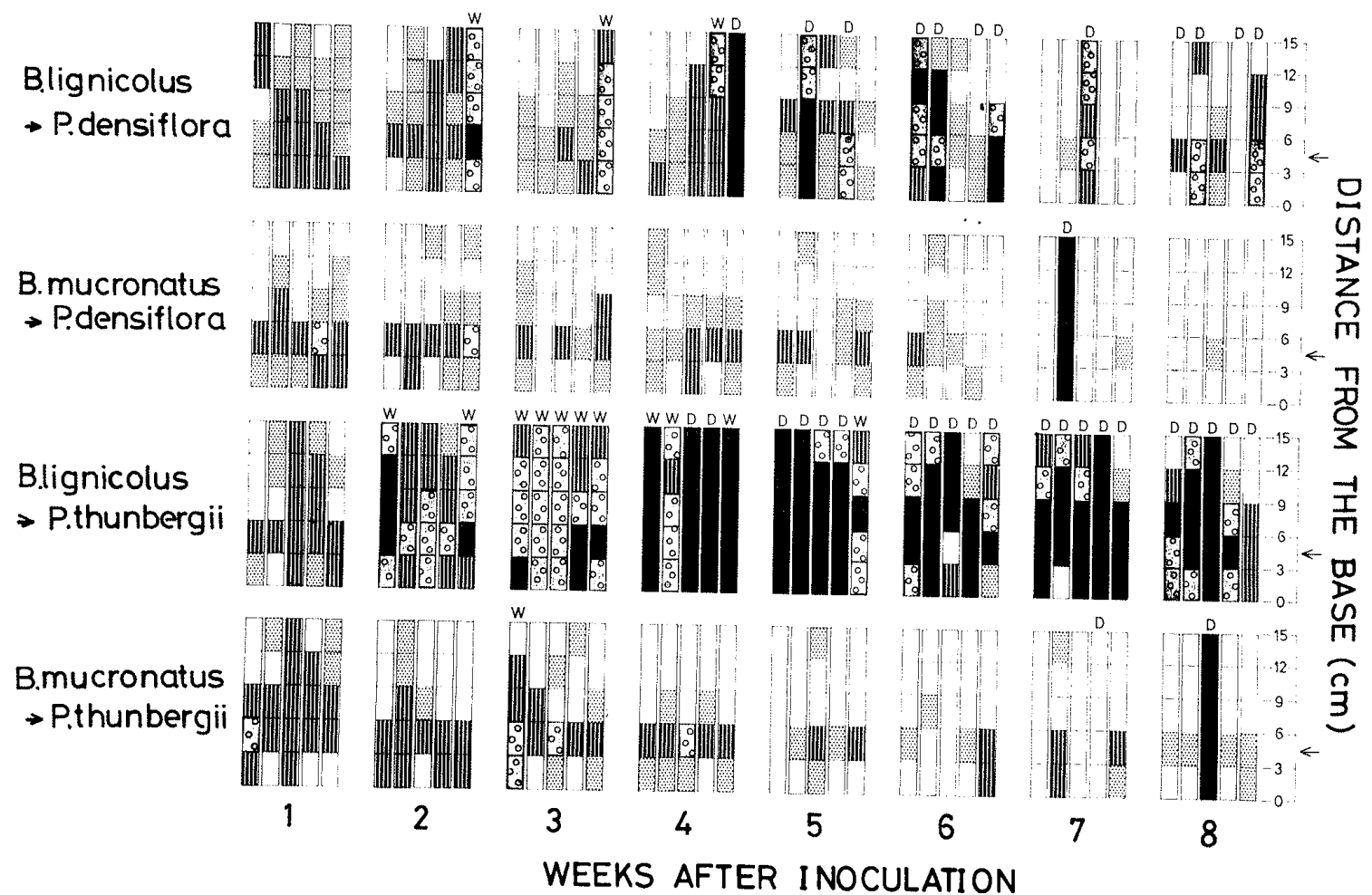

Fig. 2. Changes in distribution and population density of $B$. lignicolus and B. mucronatus in stems of inoculated seedlings.

Nematode density* is shown in five grades as follows, $\square$ : $0, \quad \square$ : $0-9$,

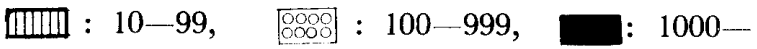

Arrows means the inoculation points. (*: nematode number recovered from each stem piece of $3 \mathrm{~cm}$ length)

dispersion and full propagation of nematodes inoculated. Figure $3 a, b$ show that population density of $B$. lignicolus increased with the lowering of water content in the stem of $P$. thunbergii, but when water content dropped to 20 to $40 \%$, population density began to decrease.

In the stem of $P$. densiflora, $B$. lignicolus rapidly dispersed as in $P$. thunbergii, but its propagation was often suppressed and the population density greatly differed from seedling to seedling.

In any seedling of $P$. thunbergii and of $P$. densiftora, $B$. mucronatus dispersed in a similar manner to $B$. lignicolus but failed to propagate in most of such seedlings resulting in gradual decrease in number. Only two seedlings, one each of $P$. thunbergii and $P$. densiflora allowed propagation of $B$. mucronatus, and these seedlings were dead. Another seedling of $P$. thunbergii inoculated with $B$. mucronatus was found to be dead at the 7th week (Fig. 2), but no nematodes were recovered from it.

\section{DISGUSSION}

The rate of nematode reproduction in a particular host is determined by various factors concerned in the relationship between the nematode and the plant. Avail- 

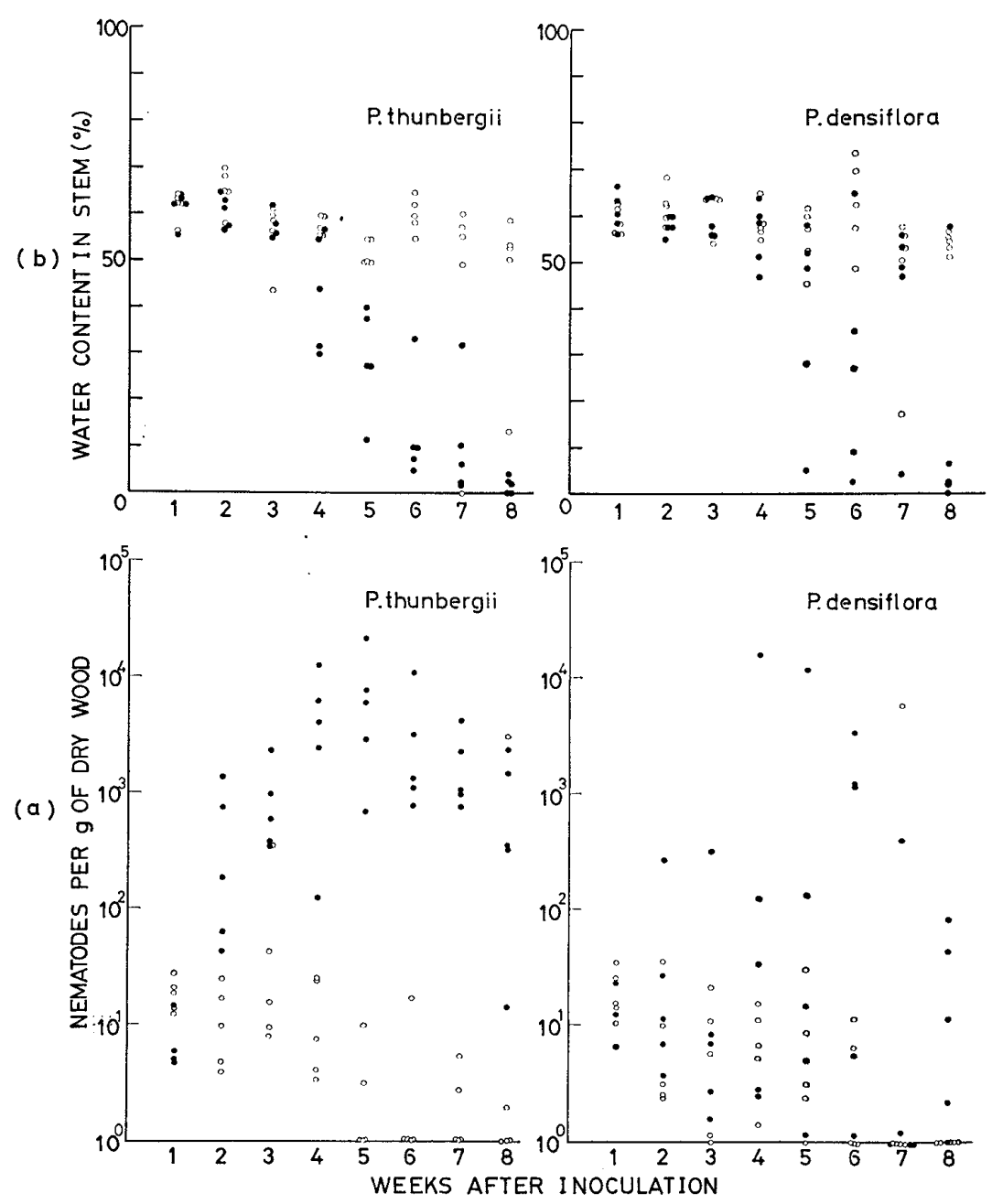

Fig. 3. (a): Population change of B. lignicolus (O) and B. mucronatus (O) in the stems of $P$. thunbergii and $P$. densiflora, and (b): changes of water content in stems after inoculation of nematodes. Every seedling of both pine species was inoculated with about 2,000 nematodes and the numbers of extracted nematodes per gramme dry weight of wood at each harvest time are presented.

ability of food, its quality, and the ability of the parasite to secure and ingest it may affect the rate of reproduction (NUSBAUM and BARKER, 1971). The reproductive rates of $B$. lignicolus on different substrates, i.e. cultured fungus (Botrytis cinerea), callus tissues of alfalfa or pine, and seedlings of pines in the field are plotted on the same graph (Fig. 4). The data on propagation of the nematode on callus tissues in this figure were quoted from the results of TAmura and Mamiya $(1975,1976)$. This figure shows that the reproductive rate of $B$. lignicolus in pine seedlings is more similar to that on callus tissues than to that on fungus culture (Botrytis cinerea). On the other hand, B. mucronatus, which can reproduce on Botrytis cinerea with a reproductive rate slightly lower than B. lignicolus (FUTAI, 1980) could not propagate on pine seedlings. Reproductive rate of $B$. lignicolus in $P$. densiflora greatly differed individually, but was lower on the average than that in $P$. thunbergii (Fig. 3). This seems to be in rela- 


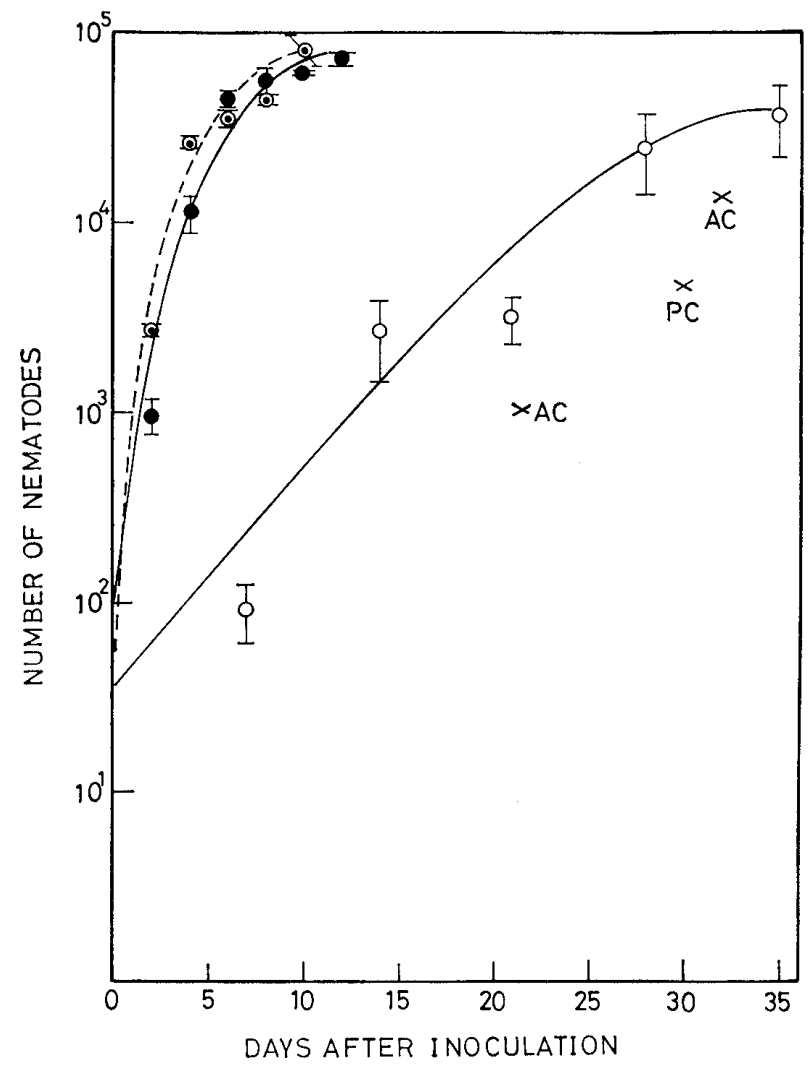

Fig. 4. Population growth of B. lignicolus on various substrates, i.e., cultured fungus Botrytis cinerea (at $29^{\circ} \mathrm{G}:----\odot$ at $\left.26^{\circ} \mathrm{G}:--\bigcirc\right)$, callus tissues of alfalfa $(\times \mathrm{AC})$ or of $P$. densiflora $(\times \mathrm{PC})$, and seedlings in the field $(P$. thunbergii: $\mathrm{O}-\mathrm{O})$.

tion with the results that the symptoms advanced more slowly in $P$. densiflora than in $P$. thunbergii (Fig. 1), and might be in support to the suggestion that $P$. densiftora has a slightly higher resistance to $B$. lignicolus than $P$. thunbergii (OHyama et al. 1974; Ohba et al. 1977; Futai and Furuno, 1979). Mamiya (1974) reported that $B$. lignicolus can propagate in seedlings of $P$. densiflora and in those of $P$. thunbergii at a similar rate, but the present data with $P$. densiflora slightly differed from MAMIYA's. While the cause of such difference is unknown, the fact that the inoculation density used here (2,000/seedling) was lower than that used by MAmiYA (5,000/seedling) might be taken into consideration.

Whether or not endoparasitic nematodes such as $B$. lignicolus can disperse in host plant seems to be another important factor related to the resistance of host. НАsнimoto and Dosono (1975) found that $B$. lignicolus rapidly dispersed in the stem of $P$. thunbergii and $P$. densiflora but its dispersion was apt to be suppressed in $P$. taeda. Results obtained in the present study also showed rapid dispersion of $B$. lignicolus in seedlings of $P$. thunbergii and $P$. densiflora. The early dispersion of $B$. mucronatus in such seedlings was similar to that of $B$. lignicolus. 


\section{AGKNOWLEDGEMENT}

I wish to express my sincere thanks to Professor A. TAkimoto, Kyoto University, for his invaluable advice and critical reading of this manuscript. Thanks are also due to Associate Professor T. Furuno and Professor S. Iwao, Kyoto University, for their helpful criticism, and to Drs. S. Kontani, K. Mineo, and K. TANAKA, Kansai Branch of Forest and Forest products Research institute, for their encouragement and suggestions in all aspects of this study.

\section{REFERENGES}

FUTAI, K. (1980) Developmental rate and population growth of Bursaphelenchus lignicolus (Nematoda: Aphelenchoididae) and B. mucronatus. Appl. Ent. Zool. $15: 115-122$.

FutaI, K. and T. Furuno (1979) The variety of resistances among pine-species to pine wood nematode, Bursaphelenchus lignicolus. Bull. Kyoto Univ. For. 51 : 23-36 (in Japanese with an English summary).

Hashimoto, H. and Y. Dosono (1975) Dispersion and reproduction of Bursaphelenchus lignicolus in resistant or in susceptible pines. Trans. Mtg. Jap. For. Soc. 86 : 301-302 (in Japanese). ${ }^{1}$

Jones, F. G. W. (1956) Soil populations of beet eelworm (Heterodera schachtii Schm.) in relation to cropping. Ann. appl. Biol. $44: 25-56$.

Mamiya, Y. (1974) Population increase of Bursaphelenchus lignicolus in wood of pine seedlings in relation with the time after inoculation. Trans. Mtg. Jap. For. Soc. $85: 249-251$ (in Japanese).

Nusbaum, G. J. and K. R. Barker (1971) Population dynamics. in Plant Parasitic Nematodes (ed. Zuckermann, B. M., W. F. Mai, and R. A. Rohde) New York: Academic Press, Vol. I, 345 pp.

Oнba, K., K. Nishimura and T. Toda (1977) Difference in survival rates among various pine races inoculated with pine wood nematodes (Bursaphelenchus lignicolus). Trans. Mtg. Kyushu Branch Jap. For. Soc. 30: 69-70 (in Japanese). ${ }^{1}$

Ohyama, N., K. Kawanobe and A. Saito (1974) The resistance of Pinus densiflora, $P$. thunbergii, $P$. taeda and $P$. elliottii to pine wood nematode (Bursaphelenchus lignicolus). Trans. Mtg. Kyushu Branch Jap. For. Soc. $27: 77-78$ (in Japanese).$^{1}$

PARker, J. (1957) Seasonal changes in some chemical and physical properties of living cells of Pinus ponderosa and their relation to freezing resistance. Protoplasma $48: 147-163$.

SeinHorst, J. W. (1967) The relationship between population increase and population density in plant parasitic nematodes. (III), (IV) Nematologica $13: 429-442$.

Tamura, H. and Y. Mamiya (1975) Reproduction of Bursaphelenchus lignicolus on alfalfa callus tissues. Nematologica 21 : 449-45 4.

TAmURA, H. and Y. MAmiYa (1976) Reproduction of Bursaphelenchus lignicolus on pine callus tissues. Trans. Mtg. Jap. For. Soc., 86: 229-230 (in Japanese).

VANGundy, S. D. and J. D. KirkPatrick (1964) Nature of resistance in certain citrus rootstocks to citrus nematode. Phytopathology $54: 419-427$.

Title translated by the present author, 